\title{
Análise da estabilidade oxidativa por termogravimetria (TGA) do sebo bovino e óleo visceral de Tilápia para produção de biodiesel de $2^{\circ}$ geração
}

\author{
L. E. OLIVEIRA ${ }^{1}$ e M. L. C. P. da SILVA ${ }^{2}$ \\ ${ }^{1}$ Universidade de São Paulo, Departamento de Biotecnologia Industrial \\ ${ }^{2}$ Universidade de São Paulo, Departamento de Engenharia Química \\ E-mail para contato: levi@ dequi.eel.usp.br
}

\begin{abstract}
RESUMO - Para o armazenamento da matéria-prima do biodiesel (óleos vegetais e gordura animal) as característica mais importantes são a estabilidade térmica e oxidativa. Nesse estudo foi realizado a termogravimetria (TGA) com a metodologia matemática Coats-Redfern do sebo bovino e óleo visceral de Tilápia para encontrar a estabilidade térmica dessas matérias primas para produção do biodiesel de segunda geração. Sabe-se que o sebo bovino participa em torno de $20 \%$ na fonte de matéria prima para produção de biodiesel no Brasil, perdendo apenas para o óleo de soja que produz o biodiesel de primeira geração. Para isso é necessário encontrar outras fontes para a produção de biodiesel de $2^{\circ}$ geração. Para o sebo bovino foi encontrado energia de ativação $\mathrm{E}=54,65$ $\mathrm{kJ} \mathrm{mol}{ }^{-1}$, entalpia de ativação $\Delta \mathrm{H}=58,08 \mathrm{~kJ} \mathrm{~mol}^{-1}$, entropia de ativação $\Delta \mathrm{S}=-117,43$ $\mathrm{J} \mathrm{mol}^{-1} \mathrm{~K}^{-1}$, energia de Gibbs de ativação $\Delta \mathrm{G}=106,46 \mathrm{~kJ}^{-1} \mathrm{~mol}^{-1}$, Fator pré-exponencial $A=6,29 \times 10^{6} \mathrm{~s}^{-1}$. Para o óleo de Tilápia foi encontrado energia de ativação $\mathrm{E}=318,91$ $\mathrm{kJ} \mathrm{mol}{ }^{-1}$, entalpia de ativação $\Delta \mathrm{H}=324,63 \mathrm{~kJ} \mathrm{~mol}^{-1}$, entropia de ativação $\Delta \mathrm{S}=231,44$ $\mathrm{J} \mathrm{mol}^{-1} \mathrm{~K}^{-1}$, energia de Gibbs de ativação $\Delta \mathrm{G}=165,17 \mathrm{~kJ} \mathrm{~mol}^{-1}$, Fator pré-exponencial $\mathrm{A}=1,76 \times 10^{25} \mathrm{~s}^{-1}$. Os resultados mostram que o óleo visceral de Tilápia apresenta maior estabilidade térmica.
\end{abstract}

\section{INTRODUÇÃ̃O}

A maior fonte para produção de biodiesel no Brasil é óleo de soja com $76 \%$ de participação seguido do sebo bovino com 20\% (ANP, 2014). Sendo esse último considerado biodiesel de segunda geração por utilizar material de descarte e não utilizar grandes extensões de terras agricultáveis. Por isso seia importante um maior incentivo em pesquisa em outras fontes para produção desse tipo de biodiesel. Além do sebo bovino, podemos destacar o óleo visceral de Tilápia que pode ser obtido facilmente em regiões pesqueiras por ser material de descarte e ainda não é aproveitado para fazer biodiesel. Esses peixes são os mais cultivados no Brasil, correspondendo a $38 \%$ do total de peixes produzidos. As vísceras representam de 7,5\% a 15\% do peso do corpo dos peixes e contêm de $35 \%$ a $45 \%$ de óleo que pode ser usado para produzir biodiesel (Santos et al., 2010). 
A estabilidade do biodiesel e suas matérias primas óleos e gorduras são uma das mais importantes propriedades em relação à sua estocagem e pode ser dividido em três categorias: Estabilidade térmica, estabilidade oxidativa e estabilidade de armazenamento (Siddharta \& Sharma, 2012). Os óleos vegetais são substâncias vulneráveis ao processo de oxidação e sua resistência aos processos oxidativos determinam a sua estabilidade oxidativa que é definida como a resistência da amostra à oxidação. Este é um parâmetro utilizado para avaliar a qualidade de óleos e gorduras e não depende apenas da composição química, mas também reflete as condições de manuseio, processamento e estocagem do produto (Garcia et al., 1993). A manutenção da integridade do biodiesel dependerá do desenvolvimento de tecnologias que aumentem sua estabilidade à oxidação durante longos tempos de estocagem.

A análise térmica, especialmente a termogravimetria (TGA) e calorimetria exploratória diferencial (DSC), tem sido utilizada para a caracterização de óleos vegetais e gorduras, estudando várias propriedades: Como a estabilidade termo-oxidativa (Buza's et al., 2000; Magoshi et al, 2003), o calor específico (Kowalski, 1988), a energia de ativação de decomposição (Kowalski, 1991), a temperatura e a entalpia de cristalização (Tan C. P. \& Man Y. B., 1999; Relkin et al., 2003; Aktas N. \& Kaya M., 2001), o efeito de antioxidantes na estabilidade térmica de óleos, o grau de cristalização (Tan P. \& Man B., 2000) e a oxidação medida por um período de indução (Gimzewski E, 1990).

O objetivo do presente trabalho é estudar a estabilidade oxidativa do sebo bovino e óleo visceral de Tilápia utilizando a termogravimetria (TGA). Além disso serão encontrados os parâmetros cinéticos e termodinâmicos da degradação termo-oxidativa utilizando a metodologia matemática Coats-Redfern.

\section{MATERIAIS E MÉTODOS}

\subsection{Amostras}

A amostra de sebo bovino foi gentilmente doada pela empresa Cognis/Jacareí. Para verificar a composição de ácidos graxos (AG) do sebo bovino foi utilizado o cromatografo gasoso Varian modelo CP3800. Enquanto que a composição de AG do óleo de tilápia foi retirado do estudo realizado por Santos et al., 2010.

Tabela 1 - Composição de ácidos graxos do sob bovino e óleo visceral de Tilápia.

\begin{tabular}{|c|c|c|}
\hline Ácidos graxos & Sebo bovino (\%) & Óleo de Tilápia (\%)* \\
\hline Palmítico (C16:0) & 22,9 & 15,9 \\
\hline Esteárico (C18:0) & 18,9 & - \\
\hline Oleico (C18:1) & 34,9 & 12,1 \\
\hline Eicosapentaenóico (C20:5) & - & 18,1 \\
\hline Docosaexaenoico (C22:6) & - & 15,8 \\
\hline Outros & 23,3 & 38,1 \\
\hline
\end{tabular}

* Fonte: Francisco et al. (2010). 


\subsection{Termogravimetria (TGA)}

Para as análises de estabilidade termo-oxidativa utilizou-se a Termobalança Shimadzu modelo TGA-50, no intervalo de temperatura de 30 a $600{ }^{\circ} \mathrm{C}$ na taxa de aquecimento de $10{ }^{\circ} \mathrm{C} \mathrm{min}^{-1}$, em cadinho de platina aberto, com atmosfera de ar sintético. Foram utilizadas amostras com massas em torno de $7,0 \mathrm{mg}$.

\subsection{Estudo cinético pelo método Coats-Redfern}

Coats e Redfern (1964) desenvolveram um método integral, que pode ser aplicada aos dados de TGA / DTG, assumindo as diferentes ordens de reação. Presume-se que a ordem relacionada com o mecanismo mais apropriado para levar a melhor regressão linear é o modelo $\mathrm{g}(\alpha)$, a partir da qual a energia de ativação é determinada. Equação 1 mostra a equação (Coats-Redfern, 1964).

$$
\ln \frac{g(\alpha)}{T^{2}}=\ln \left[\frac{A R}{\phi E}\left(1-\frac{2 R T}{E}\right)\right]-\frac{E}{R T}
$$

Onde: $\mathrm{T}=$ temperatura absoluta, $\phi=$ taxa de aquecimento $\left({ }^{\circ} \mathrm{C} \min ^{-1}\right), \mathrm{T}^{2}=$ temperatura do pico da curva DTG $\left({ }^{\circ} \mathrm{C}\right)$.

\subsection{Estudo termodinâmico}

Os parâmetros termodinâmicos foram encontrados utilizando equações matemáticas assim como Faria e Prado (2007) fizeram para estudar a degradação térmica de celulose: Equação 2 (Madhu, 2003), equação 3 (Gabal, 2003) e equação 4 (Madhu, 2003):

$$
\begin{aligned}
& E=\Delta H-R \cdot T_{s} \\
& A=\frac{K \cdot T_{s}}{h} \cdot e^{\frac{\Delta S}{R}} \\
& \Delta G=\Delta H-T_{s} \cdot \Delta S
\end{aligned}
$$

Onde, $\mathbf{k}=1,3806488 \times 10^{-23} \mathrm{~m}^{2} \mathrm{~kg} \mathrm{~s}^{-2} \mathrm{~K}^{-1}$ (constante de Boltzmann), $\mathbf{h}=6,62606957 \times 10^{-34} \mathrm{~m}^{2} \mathrm{~kg} \mathrm{~s}^{-1}$ (constante de Planck), Ts é a temperatura relacionada ao valor do pico da DTG $\left({ }^{\circ} \mathrm{C}\right)$ obtido experimentalmente, $\boldsymbol{E}$ é a energia de ativação $\left(\mathrm{kJ} \mathrm{mol}^{-1}\right), \mathbf{A}$ é o fator pré-Exponencial $\left(\mathrm{s}^{-1}\right), \Delta \mathrm{H}$ é a entalpia de ativação $\left(\mathrm{kJ} \mathrm{mol}^{-1}\right), \Delta \mathrm{S}$ é a entropia de ativação $\left(\mathrm{J} \mathrm{mol}^{-1} \mathrm{~K}^{-1}\right)$ e $\Delta \mathrm{G}$ é a energia de Gibbs de ativação $\left(\mathrm{kJ} \mathrm{mol}^{-1}\right)$. 


\section{RESULTADOS E DISCUSSÕES}

As Figuras 1 e 2 mostram as curvas obtidas por TGA do sebo bovino e óleo visceral de Tilápia. A partir dos três resultados obtidos das curvas TGA, intervalo da etapa de degradação térmica, taxa de perda massa $(\mathrm{g}(\alpha))$ e pico da DTG, foi possível encontrar os parâmetros cinéticos e termodinâmicos da degradação térmica de cada amostra utilizando a metodologia Coats-Redfern.

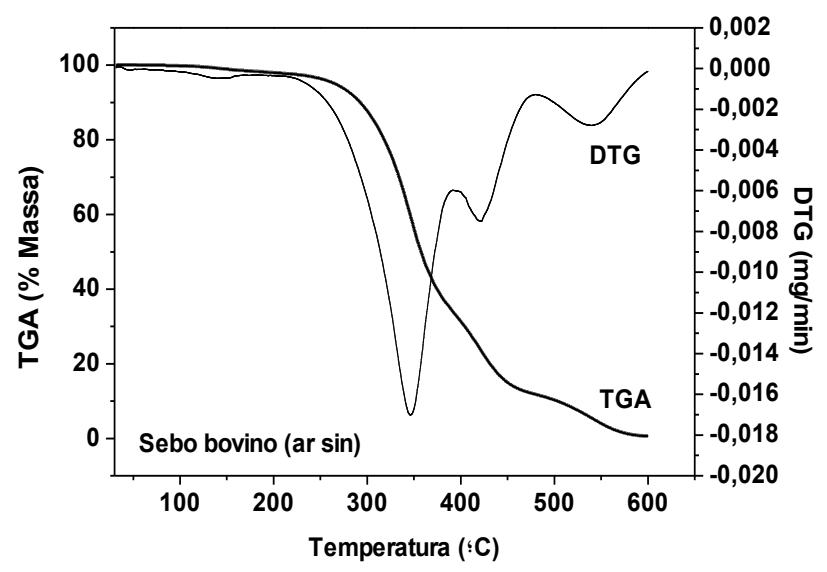

Figura 1. Curva TGA/DTG do sebo bovino.

A primeira etapa ocorre no intervalo de temperatura de 107 a $236{ }^{\circ} \mathrm{C}$, com pico da DTG igual a $139^{\circ} \mathrm{C}$ e $3 \%$ de perda de massa. Portanto a temperatura de início de degradação térmica $\left(\mathrm{T}_{\text {onset }}\right)$ é igual a $107^{\circ} \mathrm{C}$.

A segunda etapa ocorre no intervalo de temperatura de 304 a $367^{\circ} \mathrm{C}$, com o pico da DTG igual a $346{ }^{\circ} \mathrm{C}$ e $64 \%$ de perda de massa. A terceira etapa ocorre no intervalo de temperatura de 404 a $445{ }^{\circ} \mathrm{C}$, com o pico da DTG igual $422{ }^{\circ} \mathrm{C}$, com $21 \%$ de perda de massa. A quarta etapa ocorre no intervalo de temperatura de 507 a $570{ }^{\circ} \mathrm{C}$, com o pico da DTG igual a 539 ${ }^{\circ} \mathrm{C}$, com $14 \%$ de perda de massa.

O estudo cinético da degradação térmica encontrou para a primeira etapa de degradação a ordem da reação $\mathrm{n}=1,9300$, coeficiente de correlação $\mathrm{r}=0,9916$, energia de ativação $\mathrm{E}=54,6572 \mathrm{~kJ} \mathrm{~mol}^{-1}$, entalpia de ativação $\Delta \mathrm{H}=58,0826 \mathrm{~kJ} \mathrm{~mol}^{-1}$, entropia de ativação $\Delta \mathrm{S}=-117,4373 \mathrm{~J} \mathrm{~mol}^{-1} \mathrm{~K}^{-1}$, energia de Gibbs de ativação $\Delta \mathrm{G}=106,4667 \mathrm{~kJ} \mathrm{~mol}^{-1}$, Fator préexponencial $\mathrm{A}=6,2981 \times 10^{6} \mathrm{~s}^{-1}$

Para a segunda etapa de degradação foi encontrado a ordem da reação $n=1,2500$, coeficiente de correlação $\mathrm{r}=0,9993$, energia de ativação $\mathrm{E}=212,0501 \mathrm{KJ} \mathrm{mol}^{-1}$, entalpia de ativação $\Delta \mathrm{H}=217,1965 \mathrm{~kJ} \mathrm{~mol}^{-1}$, entropia de ativação $\Delta \mathrm{S}=108,7396 \mathrm{~J} \mathrm{~mol}^{-1} \mathrm{~K}^{-1}$, energia de Gibbs de ativação $\Delta \mathrm{G}=149,8867 \mathrm{~kJ} \mathrm{~mol}^{-1}$, Fator pré-exponencial $\mathrm{A}=6,1758 \mathrm{e}^{+18} \mathrm{~s}^{-1}$.

Para a terceira etapa de degradação foi encontrado a ordem da reação $n=2,0700$, coeficiente de correlação $\mathrm{r}=0,9976$, energia de ativação $\mathrm{E}=518,2225 \mathrm{KJ} \mathrm{mol}^{-1}$, entalpia de

[L1] Comentário:
[L2R1] Comentário:
[L3R1] Comentário:


ativação $\Delta \mathrm{H}=524,0008 \mathrm{~kJ} \mathrm{~mol}^{-1}$, entropia de ativação $\Delta \mathrm{S}=515,3453 \mathrm{~J} \mathrm{~mol}^{-1} \mathrm{~K}^{-1}$, energia de Gibbs de ativação $\Delta \mathrm{G}=165,8358 \mathrm{~kJ} \mathrm{~mol}^{-1}$, Fator pré-exponencial $\mathrm{A}=1,2041 \times 10^{40} \mathrm{~s}^{-1}$.

Para a quarta etapa de degradação foi encontrado a ordem da reação $n=1,7700$, coeficiente de correlação $\mathrm{r}=0,9981$, energia de ativação $\mathrm{E}=22,7505 \mathrm{KJ} \mathrm{mol}^{-1}$, entalpia de ativação $\Delta \mathrm{H}=429,5014 \mathrm{~kJ} \mathrm{~mol}^{-1}$, entropia de ativação $\Delta \mathrm{S}=286,7691 \mathrm{~J} \mathrm{~mol}^{-1} \mathrm{~K}^{-1}$, energia livre de Gibbs de ativação $\Delta \mathrm{G}=196,6449 \mathrm{~kJ} \mathrm{~mol}^{-1}$, Fator pré-exponencial $\mathrm{A}=1,6151 \times 10^{28} \mathrm{~s}^{-1}$.

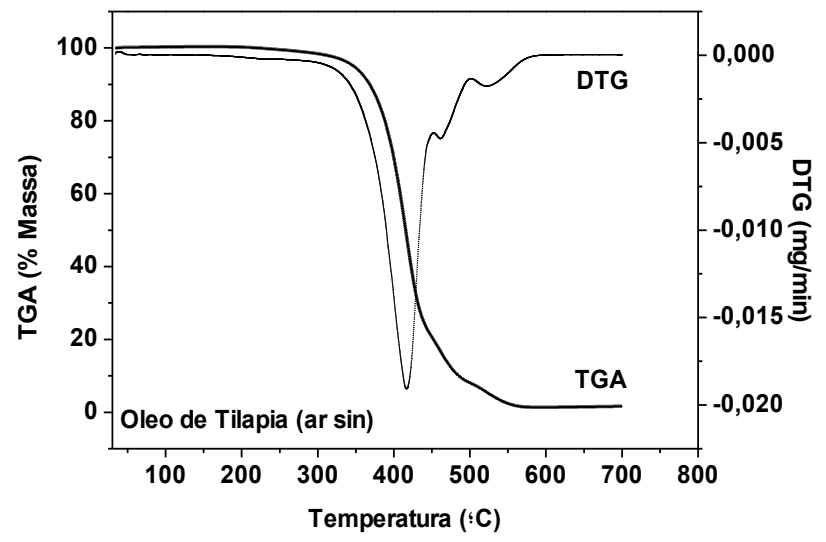

Figura 2. Curva TGA/DTG do óleo visceral de Tilápia.

O óleo visceral de Tilápia apresenta três etapas de degradação oxidativa. A primeira etapa de degradação ocorre no intervalo de temperatura de 379 a $431{ }^{\circ} \mathrm{C}$, com o pico da DTG igual a $416^{\circ} \mathrm{C}$ e $80,0 \%$ de perda de massa. Portanto o $\mathrm{T}_{\text {onset }}$ é igual a $379^{\circ} \mathrm{C}$.

A segunda etapa ocorre no intervalo de 431 a $480{ }^{\circ} \mathrm{C}$, com o pico da DTG igual a $460{ }^{\circ} \mathrm{C}$ e perda de massa de $12 \%$. A terceira e última etapa de degradação ocorre no intervalo de temperatura de 503 a $549^{\circ} \mathrm{C}$ apresentando uma perda de massa $6,0 \%$.

O estudo cinético da degradação térmica encontrou para a primeira etapa de degradação a ordem da reação $n=1,2300$, coeficiente de correlação $r=0,9990$, energia de ativação $E=318,9114$ $\mathrm{KJ} \mathrm{mol}^{-1}$, entalpia de ativação $\Delta \mathrm{H}=324,6398 \mathrm{~kJ} \mathrm{~mol}^{-1}$, entropia de ativação $\Delta \mathrm{S}=231,4469 \mathrm{~J} \mathrm{~mol}^{-1} \mathrm{~K}^{-1}$, energia de Gibbs de ativação $\Delta \mathrm{G}=165,1729 \mathrm{~kJ} \mathrm{~mol}^{-1}$, Fator pré-exponencial $\mathrm{A}=1,7662 \times 10^{25} \mathrm{~s}^{-1}$.

Para a segunda etapa de degradação foi encontrado a ordem da reação $n=2,4100$, coeficiente de correlação $r=0,9917$, energia de ativação $E=457,1955 \mathrm{KJ} \mathrm{mol}^{-1}$, entalpia de ativação $\Delta \mathrm{H}=463,2896 \mathrm{~kJ} \mathrm{~mol}^{-1}$, entropia de ativação $\Delta \mathrm{S}=402,4676 \mathrm{~J} \mathrm{~mol}^{-1} \mathrm{~K}^{-1}$, energia Gibbs $\Delta \mathrm{G}=168,2809$ $\mathrm{kJ} \mathrm{mol}^{-1}$, Fator pré-exponencial $\mathrm{A}=1,6123 \times 10^{34} \mathrm{~s}^{-1}$. 
Para a terceira etapa de degradação foi encontrado a ordem da reação $n=2,0400$, coeficiente de correlação $\mathrm{r}=0,9978$, energia de ativação $\mathrm{E}=608,8208 \mathrm{KJ} \mathrm{mol}^{-1}$, entalpia de ativação $\Delta \mathrm{H}=615,4138 \mathrm{~kJ} \mathrm{~mol}^{-1}$, entropia de ativação $\Delta \mathrm{S}=532,9192 \mathrm{~J} \mathrm{~mol}^{-1} \mathrm{~K}^{-1}$, energia de Gibbs de ativação $\Delta \mathrm{G}=192,8089 \mathrm{~kJ} \mathrm{~mol}^{-1}$, Fator pré-exponencial $\mathrm{A}=1,1375 \times 10^{41} \mathrm{~s}^{-1}$.

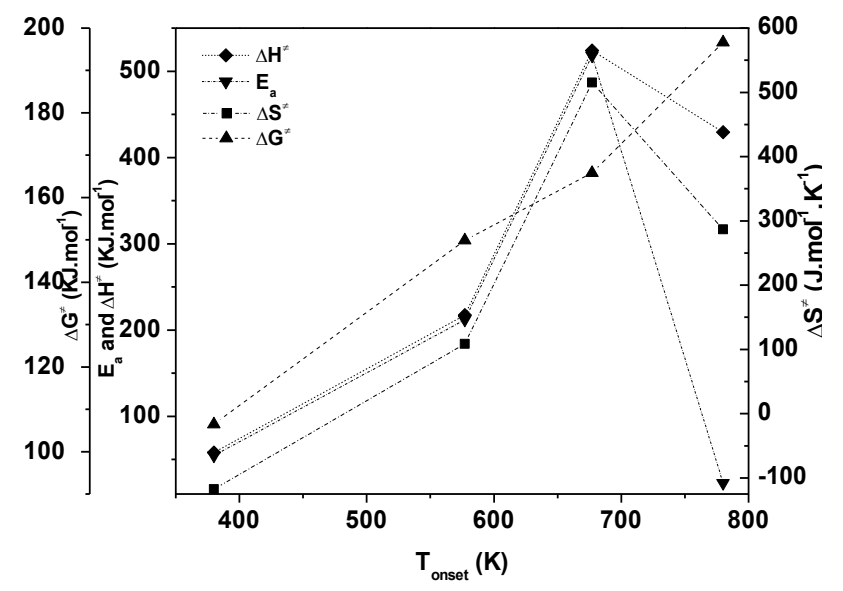

Figura 3. Parâmetros cinéticos e termodinâmicos do sebo bovino (4 etapas).

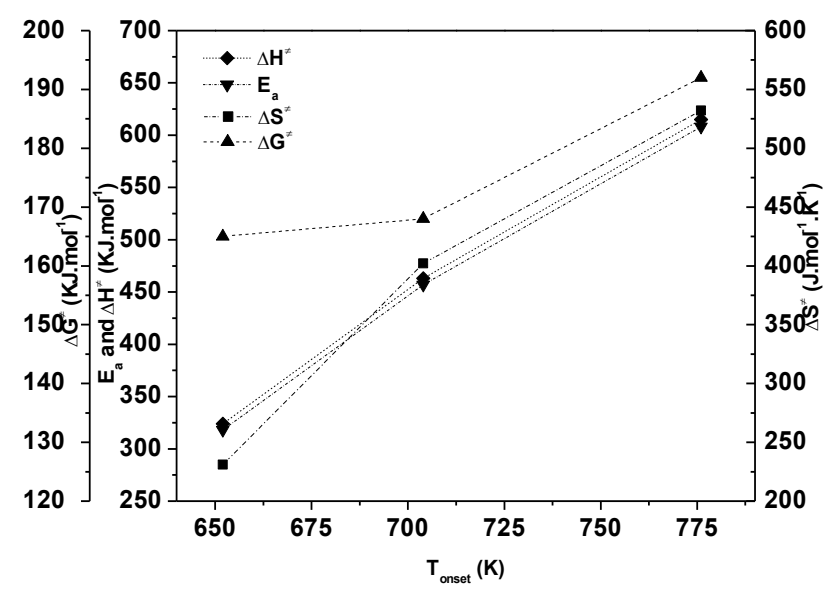

Figura 4. Parâmetros cinéticos e termodinâmicos do óleo visceral de Tilápia (3 etapas). 
A energia de ativação $(\mathrm{E})$ e temperaturas de início de degradação térmica $\left(\mathrm{T}_{\text {onset }}\right)$ mostram que o sebo bovino apresenta menor estabilidade térmica que o óleo visceral de Tilápia. Mostrando que a matéria-prima que apresenta maior quantidade de ácidos graxos (AG) de cadeias maiores, apresentará maior estabilidade térmica. Além disso, verifica-se que nas primeiras três etapas de degradação de ambas as amostras ocorrem um aumento nos parâmetros cinéticos e termodinâmicos. Em relação à quarta etapa de degradação térmica do sebo bovino, houve uma diminuição desses parâmetros visto se tratar de uma etapa que ocorre em um menor intervalo de temperatura comparado com outras etapas.

\section{CONCLUSÕES}

O estudo da estabilidade térmica por TGA do sebo bovino e do óleo visceral de Tilápia mostrou que o óleo de Tilápia apresenta maior estabilidade térmica devido à uma maior quantidade AG. No entanto, são necessários mais estudos em relação à estabilidade oxidativa dessas duas amostras visto que a estabilidade térmica pode estar bastante relacionado ao tamanho da cadeia dos ácidos graxos, enquanto que resultados obtidos da estabilidade oxidativa podem mostrar que o óleo visceral de Tilápia apresente menor estabilidade devido à uma maior quantidade de insaturações presentes na cadeia carbônica dos AGs.

\section{AGRADECIMENTOS}

Ao Cnpq pelo apoio financeiro.

\section{REFERÊNCIAS}

ANP, BOLETIM MENSAL DO BIODIESEL, (http://www.anp.gov.br/?pg= 70942\&m $=\& \mathrm{t} 1=\& \mathrm{t} 2=\& \mathrm{t} 3=\& \mathrm{t} 4=\& \mathrm{ar}=\& \mathrm{ps}=\&$ cachebust $=1404304797700)$.

AKTAS, N., KAYA, M. Detection of beef body fat and margarine in butterfat by differential scanning calorimetry. J Therm. Anal. Calorim., v. 66, p. 795-801, 2001.

BUZA'S I, SIMON J., HOLLO J., Study of the thermooxidative behavior of edible oils by thermal analysis. J Am Oil Chem Soc 1979;56:685-8.

FARIA, E. A., PRADO, A. G. S. Kinetic studies of the thermal degradation of cellulose acetate/niobium and chitosan/niobium composites. Reactive \& Functional Polymers, v. 67, p. 655-661, 2007.

GABAL, M. A. Kinetics of the thermal decomposition of. CuC. 2. O. 4. -ZnC. 2. O. 4. mixture in air. Thermochim. Acta, v. 402, p. 199-208, 2003.

GIMZEWSKI, E., A multi-sample high-pressure DTA for measuring oxidation induction times. Thermochim Acta, v. 170, p. 97-105, 1990.

RELKIN, P., SOURDT, S., FOSSEUX, P. Y. Fat crystallization in complex food emulsions. J Therm 
Anal. Calorim. v. 71, p. 187-95, 2003.

SANTOS, L.D., FURUYA, W.M., MATSUShitA, M., SIlVA, L. C. R., SILVA, T. S. D. C., BOTARO, D. Conjugated linoleic acid (CLA) in Nile tilapia diets: productive performance, chemical and fatty acids composition. Rev Bras Zootec 2007;36:1481-8.

SHARMA, B. K.; STIPANOVIC, A. J. Development of a new oxidation stability test method for lubrificating oils usin $\mathrm{g}$ high-pressure differential scanning calorimetry, Thermochim. Acta, v. 1, p. 402, 2003.

SIDDHARTA, J., SHARMA, M.P. Thermal stability of biodiesel and its blends: A review. Renewable and Sustainable Energy Reviews, v. 15, p. 438-48, 2011.

SILVA, G. A., Síntese Enzimática, Caracterização Físico-Química e Térmica De Biodiesel de Sebo Bovino por Rota Etílica. Dissertação (Mestrado) - Escola de Engenharia de Lorena -EEL-USP, Lorena, Brasil, 2009.

MADHU, N. T., RADHAKRISHAN, P. K., GRUNERT, M., WEINBERGER, P., Linert, W., Thermochim. Acta 407 (2003) 73-84.

MAGOSHI, J., BECKER, M. A., HAN Z., NAKAMURA S., Thermal properties of seed proteins. J Therm. Anal. Calorim., v. 70, p. 833-9, 2003.

TAN, C.P., MAN, Y.B., Differential scanning calorimetric analysis of edible oils: comparison of thermal properties and chemical composition. J. Am. Oil Chem. Soc., v. 77, p. 143-55, 2000.

TAN, C.P., MAN, Y.B., Differential scanning calorimetric analysis for monitoring the oxidation of heated oils. Food Chem., v. 67, p. 177-84, 1999. 